\title{
A KILOPARSEC-SCALE BINARY ACTIVE GALACTIC NUCLEUS CONFIRMED BY THE EXPANDED VERY LARGE ARRAY
}

\author{
Hai Fu ${ }^{1}$, Zhi-Yu Zhang ${ }^{2,3,4}$, Roberto J. Assef ${ }^{5,11}$, Alan Stockton ${ }^{6}$, Adam D. Myers ${ }^{7,8}$, Lin Yan ${ }^{9}$, S. G. Djorgovski ${ }^{1,12}$, \\ J. M. Wrobel ${ }^{10}$, AND DominiK A. Riechers ${ }^{1}$ \\ ${ }^{1}$ Astronomy Department, California Institute of Technology, MS 249-17, Pasadena, CA 91125, USA \\ ${ }^{2}$ Purple Mountain Observatory, CAS, 2 West Beijing Road, Nanjing, 210008, China \\ ${ }^{3}$ Max-Planck-Institut für Radioastronomie, Auf dem Hügel 69, D-53121 Bonn, Germany \\ ${ }^{4}$ Graduate School of the Chinese Academy of Sciences, 19A Yuquan Road, P.O. Box 3908, Beijing 100039, China \\ 5 Jet Propulsion Laboratory, California Institute of Technology, MS 169-530, 4800 Oak Grove Dr., \\ Pasadena, CA 91109, USA \\ ${ }^{6}$ Institute for Astronomy, University of Hawaii, 2680 Woodlawn Drive, Honolulu, HI 96822, USA \\ ${ }^{7}$ Department of Physics and Astronomy, University of Wyoming, Laramie, WY 82071, USA \\ ${ }^{8}$ Max-Planck-Institut für Astronomie, Königstuhl 17, D-69117 Heidelberg, Germany \\ ${ }^{9}$ Spitzer Science Center, California Institute of Technology, MS 220-06, Pasadena, CA 91125, USA \\ ${ }^{10}$ National Radio Astronomy Observatory, P.O. Box O, Socorro, NM 87801, USA \\ Received 2011 August 29; accepted 2011 September 13; published 2011 September 29
}

\begin{abstract}
We report the confirmation of a kiloparsec-scale binary active galactic nucleus (AGN) with high-resolution radio images from the Expanded Very Large Array (EVLA). SDSS J150243.1+111557 is a double-peaked [O III] AGN at $z=0.39$ selected from the Sloan Digital Sky Survey. Our previous near-infrared adaptive optics imaging reveals two nuclei separated by 1".4 $(7.4 \mathrm{kpc})$, and our optical integral-field spectroscopy suggests that they are a type-1-type-2 AGN pair. However, these data alone cannot rule out the single AGN scenario where the narrow emission-line region associated with the secondary is photoionized by the broad-line AGN in the primary. Our new EVLA images at 1.4, 5.0, and $8.5 \mathrm{GHz}$ show two steep-spectrum compact radio sources spatially coincident with the optical nuclei. The radio power of the type- $2 \mathrm{AGN}$ is an order-of-magnitude in excess of star-forming galaxies with similar extinction-corrected [O II] $\lambda 3727$ luminosities, indicating that the radio emission is powered by accretion. Therefore, SDSS J150243.1+111557 is one of the few confirmed kiloparsec-scale binary AGN systems. Spectral energy distribution modeling shows that SDSS J150243.1+111557 is a merger of two $\sim 10^{11} M_{\odot}$ galaxies. With both black hole masses around $10^{8} M_{\odot}$, the AGNs are accreting at $\sim 10$ times below the Eddington limit.
\end{abstract}

Key words: galaxies: active - galaxies: individual (SDSS J150243.1+111557) - galaxies: interactions - galaxies: nuclei

\section{INTRODUCTION}

Binary supermassive black holes (SMBHs) resulting from galaxy mergers should be commonplace, given that virtually every massive galaxy harbors an SMBH (Richstone et al. 1998). However, cases are rare where both SMBHs in a merging system are accreting as active galactic nuclei (AGNs). Hundreds of binary AGNs with $>10 \mathrm{kpc}$ separations have been discovered both at high redshifts (Myers et al. 2008; Hennawi et al. 2010) and locally (Liu et al. 2011). But, there are only a few confirmed kiloparsec-scale binary AGNs (Junkkarinen et al. 2001; Komossa et al. 2003; Koss et al. 2011). Such close binaries are important, because strong tidal interactions are more likely to influence the nuclear accretion and star formation in both galaxies at kpc-scale separations (Barnes \& Hernquist 1996).

Systematic searches for kiloparsec-scale binary AGNs have focused on AGNs with pairs of narrow [O III] $\lambda 5007$ emission lines in spatially integrated spectra (Gerke et al. 2007; Comerford et al. 2009; Wang et al. 2009; Liu et al. 2010b; Smith et al. 2010), which account for $\sim 1 \%$ of the AGNs in the Sloan Digital Sky Survey (SDSS). SDSS data are inadequate to confirm such binaries because they are typically spatially unresolved in seeing-limited optical data. However, subsequent highresolution imaging and spatially resolved spectroscopy have

\footnotetext{
${ }^{11}$ NASA Postdoctoral Program Fellow.

${ }^{12}$ Distinguished Visiting Professor, King Abdulaziz University, Jeddah, Saudi Arabia.
}

found that most of these AGNs show double-peaked line profiles because of gas kinematics driven by outflows, inflows, or disk rotation (Shen et al. 2011; Fu et al. 2011b). Nonetheless, these observations have also found a few binary AGN candidates that show Seyfert-like emission-line regions that spatially coincide with the stellar nuclei of the merging galaxies (Liu et al. 2010a; McGurk et al. 2011; Fu et al. 2011b). Yet, none of these candidates has been confirmed to host two actively accreting SMBHs, because with the optical data alone, it is difficult to rule out that a Seyfert-2-type emission-line region is photoionized by an AGN outside of that region. High-resolution radio or X-ray detections of both nuclei can provide unambiguous evidence for active accretion (see the Very Long Baseline Array searches of Tingay \& Wayth 2011 and Burke-Spolaor 2011).

SDSS J150243.1+111557 $(z=0.39, r=18.0$; hereafter SDSS J1502+1115) is a double-peaked type-1 AGN identified by Smith et al. (2010). Our previous observations suggest that it is a seven-kiloparsec-separation type-1-type-2 AGN pair (Fu et al. 2011b). Here we report radio continuum imaging from the Expanded Very Large Array (EVLA; Perley et al. 2011), which confirms SDSS J1502+1115 as a binary AGN. Throughout we adopt a $\Lambda$ CDM cosmology with $\Omega_{\mathrm{m}}=0.3, \Omega_{\Lambda}=0.7$, and $H_{0}=$ $70 \mathrm{~km} \mathrm{~s}^{-1} \mathrm{Mpc}^{-1}$.

\section{OBSERVATIONS}

We observed SDSS J1502+1115 with the EVLA in the A configuration under project code 11A-175. The EVLA was 


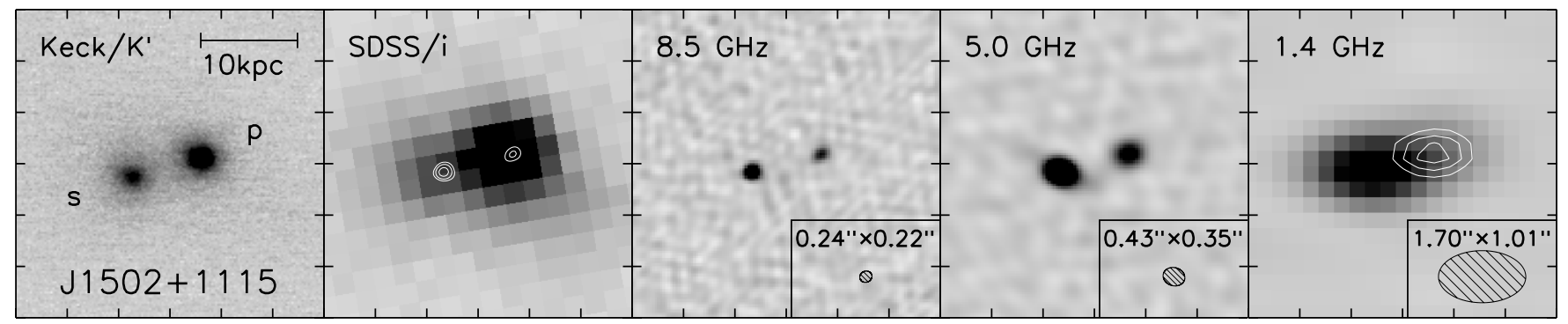

Figure 1. Keck $/ K^{\prime}$-band, SDSS $/ i$-band, and EVLA $/ X-C-L$-bands 6 " image stamps of SDSS J1502+1115. The scale bar in the $K^{\prime}$ image indicates a transverse separation of $10 \mathrm{kpc}$. The primary ("p") and secondary ("s") AGNs are labeled. The $8.5 \mathrm{GHz}$ contours in the $i$-band image are at $8 \sigma, 16 \sigma, 32 \sigma$, where $1 \sigma=$ $23 \mu \mathrm{Jy}$ beam $^{-1}$. To better illustrate the primary in the $1.4 \mathrm{GHz}$ panel, we show contours from the residual image after subtracting the secondary. The contour levels are $32 \sigma, 40 \sigma$, and $48 \sigma$, where $1 \sigma=44 \mu \mathrm{Jy}_{\text {beam }}{ }^{-1}$. Hatched ellipses at the lower right corners of the radio maps show the EVLA beams. The images are aligned based on their own astrometry and are displayed in linear scale. $\mathrm{N}$ is up and $\mathrm{E}$ is to the left. Tickmarks are spaced in $1^{\prime \prime}$.

set up with dual circular polarizations and a total bandwidth of $256 \mathrm{MHz}$ per polarization at central frequencies of 1.391 , 4.959, and $8.459 \mathrm{GHz}$ (hereafter 1.4, 5.0, 8.5 GHz). The 1.4 and $5.0 \mathrm{GHz}$ observations were carried out consecutively on 2011 June 29 (UT) and the $8.5 \mathrm{GHz}$ observations were carried out on 2011 June 14 (UT). The total on-source exposure times were 25,35 , and 20 minutes at $1.4,5.0$, and $8.5 \mathrm{GHz}$, respectively. The nearby point-like quasar J1504+1029 was used as the gain calibrator. The switching angle was $53^{\prime}$, and the switching times were 7,10 , and 10 minutes at $1.4,5.0$, and $8.5 \mathrm{GHz}$, respectively. 3C 286 was observed for bandpass and flux-density calibration.

Data calibration was carried out with the Common Astronomical Software Applications (CASA) package, while imaging employed both CASA and AIPS (Astronomical Image Processing System). For the 5.0 and $8.5 \mathrm{GHz}$ data, we used a Briggs robust parameter of 0 to achieve good image fidelity with only a modest loss of sensitivity compared to "natural" weighting. For the $1.4 \mathrm{GHz}$ data, we used "uniform" weighting to achieve the best resolution. The final images, shown in Figure 1, have rms noise levels of 44,17 , and $23 \mu \mathrm{Jy}$ beam $^{-1}$ for $1.4,5.0$, and $8.5 \mathrm{GHz}$, respectively.

SDSS J1502+1115 is resolved into two components, a primary ("p") and a secondary ("s"), separated by 1".4 at all frequencies (Figure 1). Their $8.5 \mathrm{GHz}$ centroids are at R.A. = 15:02:43.088, decl. $=+11: 15: 57.42$ (p), and R.A. $=$ 15:02:43.179, decl $=+11: 15: 57.07$ ( $;$; J2000). The secondary shows hints of adjacent structure at $5.0 \mathrm{GHz}$; to quantify this structure at $8.5 \mathrm{GHz}$, a matched-resolution image was made by excluding visibility data with baselines longer than those available at $5.0 \mathrm{GHz}$. This matched-resolution image was used for component photometry at $8.5 \mathrm{GHz}$.

The integrated flux densities of the primary and secondary at each frequency appear in Table 1, where the errors are the quadratic sum of the error in the fit and a 3\% error in the flux-density scale. The combined $1.4 \mathrm{GHz}$ flux density is $9.62 \pm 0.42 \mathrm{mJy}$, consistent with the integrated flux from the FIRST catalog ( $9.30 \pm 0.49 \mathrm{mJy}$; White et al. 1997). Both sources are compact. The beam-deconvolved sizes of both components are less than $0.3(1.6 \mathrm{kpc})$ at both 5.0 and $8.5 \mathrm{GHz}$; the size measurements from the $1.4 \mathrm{GHz}$ image are less accurate.

\section{ANALYSIS AND RESULTS}

The EVLA images are analyzed in combination with a $K^{\prime}$-band image from the Keck II laser guide star adaptive optics system (Wizinowich et al. 2006) and seeing-limited optical integral-field spectra from the Supernova IntegralField Spectrograph (SNIFS; Aldering et al. 2002) on the UH
Table 1

Spectral Energy Distribution

\begin{tabular}{|c|c|c|c|}
\hline $\begin{array}{l}\text { Band } \\
\text { (1) }\end{array}$ & $\begin{array}{c}\lambda \\
(\mu \mathrm{m}) \\
(2)\end{array}$ & $\begin{array}{c}F_{v}(\text { Primary }) \\
(\mathrm{mJy}) \\
(3)\end{array}$ & $\begin{array}{c}F_{\nu}(\text { Secondary }) \\
(\mathrm{mJy}) \\
(4)\end{array}$ \\
\hline FUV & 0.16 & \multicolumn{2}{|c|}{$0.043 \pm 0.010$} \\
\hline NUV & 0.23 & \multicolumn{2}{|c|}{$0.061 \pm 0.009$} \\
\hline$u$ & 0.36 & $0.075 \pm 0.005$ & $0.012 \pm 0.005$ \\
\hline$g$ & 0.47 & $0.110 \pm 0.003$ & $0.038 \pm 0.003$ \\
\hline$r$ & 0.62 & $0.114 \pm 0.005$ & $0.110 \pm 0.005$ \\
\hline$i$ & 0.75 & $0.164 \pm 0.026$ & $0.179 \pm 0.026$ \\
\hline$z$ & 0.89 & $0.259 \pm 0.016$ & $0.258 \pm 0.016$ \\
\hline$Y$ & 1.03 & $0.313 \pm 0.006$ & $0.272 \pm 0.006$ \\
\hline$J$ & 1.25 & $0.354 \pm 0.006$ & $0.321 \pm 0.006$ \\
\hline$H$ & 1.63 & $0.385 \pm 0.010$ & $0.448 \pm 0.010$ \\
\hline$K$ & 2.20 & $0.444 \pm 0.012$ & $0.582 \pm 0.012$ \\
\hline WISE1 & 3.35 & \multicolumn{2}{|c|}{$1.049 \pm 0.026$} \\
\hline WISE2 & 4.60 & \multicolumn{2}{|c|}{$1.538 \pm 0.038$} \\
\hline WISE3 & 11.56 & \multicolumn{2}{|c|}{$4.733 \pm 0.179$} \\
\hline WISE4 & 22.09 & \multicolumn{2}{|c|}{$18.688 \pm 1.102$} \\
\hline $8.5 \mathrm{GHz}$ & 35290.46 & $0.61 \pm 0.04$ & $1.28 \pm 0.06$ \\
\hline $5.0 \mathrm{GHz}$ & 60454.21 & $0.93 \pm 0.05$ & $2.33 \pm 0.08$ \\
\hline $1.4 \mathrm{GHz}$ & 215522.97 & $2.58 \pm 0.25$ & $7.04 \pm 0.34$ \\
\hline
\end{tabular}

Note. Because we cannot separate the two components in GALEX and WISE images, we list their total fluxes.

$2.2 \mathrm{~m}$ telescope. The $K^{\prime}$-band image resolved SDSS J1502+1115 into two components separated by 1".39 (7.4 kpc; Fu et al. 2011a), and the integral-field spectroscopy spatially resolved the kinematic components and associated them with the two stellar nuclei (Fu et al. 2011b). The [O III] line of the primary is blueshifted relative to that of the secondary by $657 \mathrm{~km} \mathrm{~s}^{-1}$. In Figure 2, we show the optical spectra of the two components extracted from the SNIFS datacube with $0{ }^{\prime \prime} 8$ apertures. The primary is a type-1 AGN with broad Mg II and Balmer emission lines along with high-ionization narrow lines, while the secondary is a type- 2 AGN with narrow high-ionization lines and line ratios that are consistent with AGN photoionization according to the Baldwin et al. (1981) diagrams (Fu et al. 2011b).

We compare the EVLA images with the SDSS $i$-band and the $K^{\prime}$-band images in Figure 1. The offset between the two radio sources and the near-infrared (NIR) sources is only $0{ }^{\prime} ! 1$, much smaller than the $1 \sigma$ dispersion $\left(\sim 0^{\prime \prime} 4\right)$ between typical SDSS and FIRST positions.

Both sources show steep radio spectra, with $\alpha=-0.80 \pm 0.06$ and $-0.92 \pm 0.04\left(F_{\nu} \propto v^{\alpha}\right)$ for the primary and secondary, respectively, indicating optically thin synchrotron emission. 


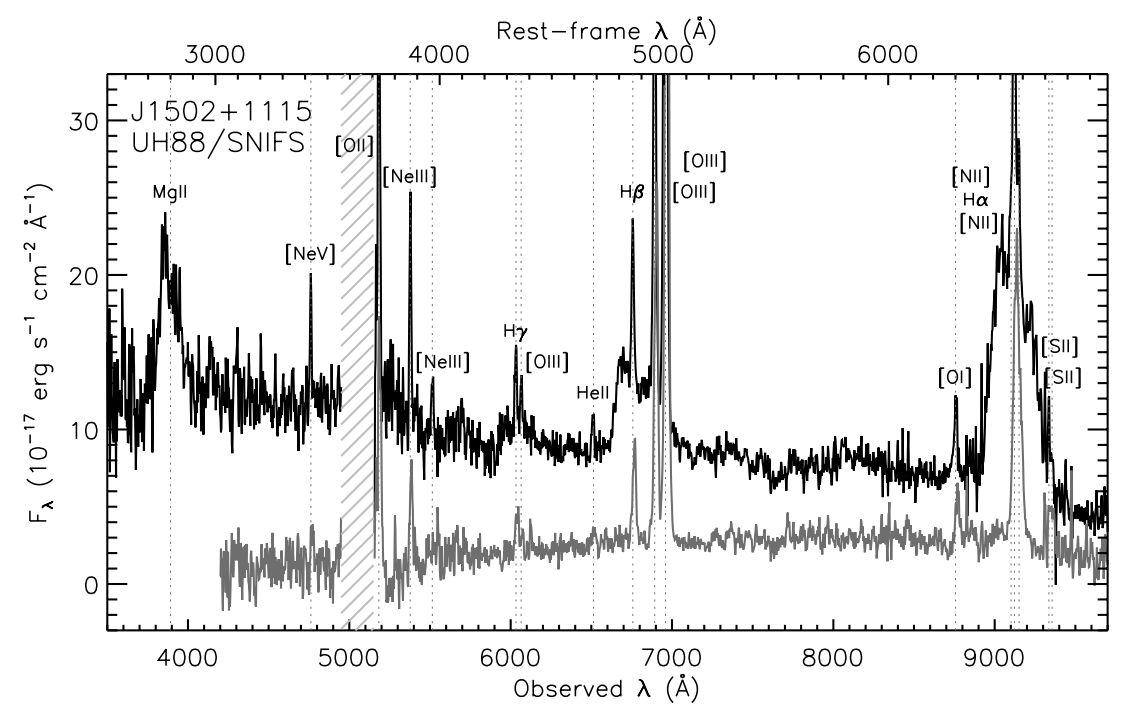

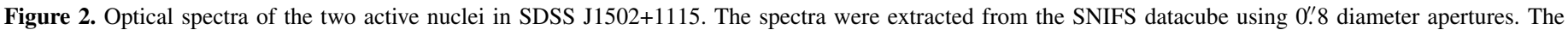

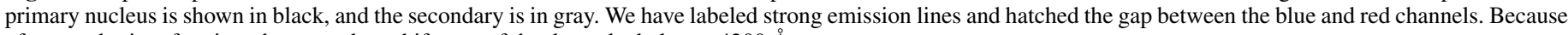
of atmospheric refraction, the secondary shifts out of the datacube below $\sim 4200 \AA$.

Compact steep-spectrum radio sources like SDSS J1502+1115 make up $\sim 30 \%$ of bright radio sources and they are mostly powerful AGNs (O'Dea 1998). Since star formation and black hole accretion are the two major powering sources of extragalactic synchrotron radiation, we consider the two scenarios in the following.

\subsection{Nature of the Radio Emission in SDSS J1502+1115}

If the radio emission is entirely driven by star formation, the rest-frame $1.4 \mathrm{GHz}$ power of $4.6 \times 10^{23}$ and $1.3 \times 10^{24} \mathrm{~W} \mathrm{~Hz}^{-1}$ implies star formation rates (SFRs) of 273 and $787 M_{\odot} \mathrm{yr}^{-1}$ for the primary and secondary, respectively (Yun et al. 2001). However, these tremendous amounts of star formation disagree with other well-established star formation indicators, such as the far-infrared (FIR) luminosity and the [O II] $\lambda 3727$ luminosity, especially for the type- 2 secondary, indicating that star formation is unlikely to dominate the radio emission.

First, SDSS J1502+1115 is undetected by IRAS in all four bands $(12,25,60$, and $100 \mu \mathrm{m})$. The scanpi ${ }^{13} 60 \mu \mathrm{m} 3 \sigma$ upper limit of $0.15 \mathrm{Jy}$ sets an upper limit of $2.2 \times 10^{23} \mathrm{~W} \mathrm{~Hz}^{-1}$ to the $1.4 \mathrm{GHz}$ power from star formation, adopting the FIR-radio correlation of Yun et al. (2001). This total upper limit is 2.0 and 5.9 times less than that observed for the primary and secondary alone.

Second, the [O II] luminosities give SFRs much less than those from the radio luminosity, even if we make the unrealistic assumption that all of the $[\mathrm{O} \mathrm{II}]$ emission comes from star formation. We use the narrow $\mathrm{H} \alpha / \mathrm{H} \beta$ ratio to estimate the intrinsic extinction, given an intrinsic ratio of 3.1 (Osterbrock $\&$ Ferland 2006). We find extinctions of $A_{V}^{p}=0.78$ and $A_{V}^{S}=0.28$. We then use Cardelli et al. (1989) extinction curve to deredden the total $[\mathrm{O}$ II $]$ fluxes $^{14}\left(f_{[\mathrm{O} \text { II }}^{p}=1.0 \times 10^{-14}\right.$ and $f_{\text {[O II] }}^{s}=7.6 \times 10^{-15} \mathrm{erg} \mathrm{s}^{-1} \mathrm{~cm}^{-2}$ ). The dereddened luminosities imply SFRs of 82 and $31 M_{\odot} \mathrm{yr}^{-1}$ (Kennicutt 1998), which are 3.3 and 26 times less than the SFRs from the $1.4 \mathrm{GHz}$ power for the primary and secondary, respectively.

\footnotetext{
${ }_{13}$ http://scanpi.ipac.caltech.edu:9000/applications/Scanpi

14 These have been corrected for Galactic extinction and aperture loss.
}

To explore whether the radio emission can be powered by black hole accretion, we can consider whether the radio emission is in significant excess of the X-ray emission when compared with typical AGNs. SDSS J1502+1115 is detected by ROSAT with an X-ray flux of $S_{0.1-2.4 \mathrm{keV}}=(4.9 \pm 1.7) \times$ $10^{-13} \mathrm{erg} \mathrm{cm}^{-2} \mathrm{~s}^{-1}\left(0.0429 \pm 0.0147\right.$ counts s$\left.{ }^{-1}\right)$. Accounting for the Galactic absorption $\left(N_{\mathrm{H}}=2.37 \times 10^{20} \mathrm{~cm}^{-2}\right)$, we estimated ${ }^{15}$ rest-frame X-ray luminosities of $L_{0.5-2 \mathrm{keV}}=(6.5 \pm$ $2.2) \times 10^{43} \mathrm{erg} \mathrm{s}^{-1}$ and $L_{2-10 \mathrm{keV}}=(1.0 \pm 0.6) \times 10^{44} \mathrm{erg} \mathrm{s}^{-1}$ for a photon index $\Gamma=1.8$. This X-ray power and the absolute restframe $B$-band magnitude $M_{\mathrm{B}}=-22.7$ place SDSS J1502+1115 on the borderline between Seyferts and quasars (Brusa et al. 2007). The rest-frame $5 \mathrm{GHz}$ luminosity of the entire system is $3.0 \times 10^{40} \mathrm{erg} \mathrm{s}^{-1}$. The $5 \mathrm{GHz}$ to hard X-ray luminosity ratio, $\log R_{\mathrm{X}}=\log \left(\nu L_{5 \mathrm{GHz}} / v L_{2-10 \mathrm{keV}}\right)=-3.5$, lies between the median values of Seyfert- $1 \mathrm{~s}\left(\log R_{\mathrm{X}}=-3.8\right)$ and Seyfert-2s ( -3.3 ; see Table 1 of Ho 2008). Therefore, most of the radio emission could be powered by accretion. The intermediate $\log R_{\mathrm{X}}$ value also hints that SDSS J1502+1115 is a type- $1-$ type- 2 binary AGN.

\subsection{Spectral Energy Distribution Modeling}

We have compiled 15-band photometry from the Galaxy Evolution Explorer (GALEX: FUV, NUV; Martin et al. 2005), SDSS DR8 ( $u, g, r, i$, and $z$; Aihara et al. 2011), UKIRT Infrared Sky Survey (UKIDSS) DR6plus $(Y, J, H$, and $K$; Lawrence et al. 2007), and WISE (3.4, 4.6, 12, and $22 \mu \mathrm{m}$; Wright et al. 2010) surveys. The primary and secondary are blended in all of these catalogs but can be deblended in the SDSS and UKIDSS images. We use GALFIT (Peng et al. 2010) to deblend the SDSS and UKIDSS images. We cannot separate the two components in the GALEX and WISE images given the small separation and their large point-spread functions (PSFs). For the GALFIT input PSF, we use a stellar source $83^{\prime \prime}$ to the southwest. For the primary, either a direct PSF or a slightly Gaussian-convolved (FWHM $\sim 1$ pixel $=$ 0.4) PSF provides a good fit. For the secondary, we used a Sérsic profile. While modeling the $g-, r-$, and $i$-band images,

\footnotetext{
15 webPIMMS: http://heasarc.nasa.gov/Tools/w3pimms.html
} 


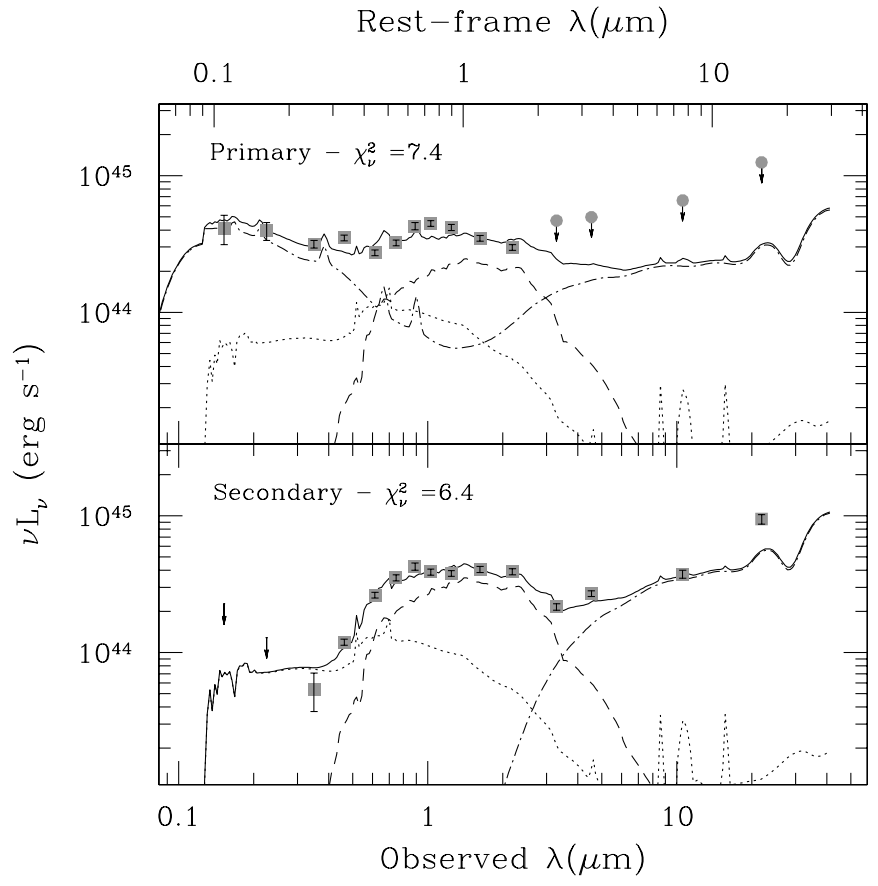

Figure 3. Best-fit SED (solid line) to the primary (top) and secondary (bottom) components of SDSS J1502+1115 using the templates of Assef et al. (2010). The best-fit SEDs are a combination of a Type-1 AGN (dot-dashed line) with an old stellar population (dashed line) and a star-forming component (dotted line). The photometry of the primary and secondary components is shown by gray solid squares. WISE fluxes could not be deblended and are assumed to be upper bounds for the primary component SED fit (gray solid circles). See Section 3.2 for details.

we also masked out areas between the two galaxies and south of the primary that are contaminated by [O II] and [O III] emission lines from the extended nebulae. For the $u_{-}, g_{-}, r-$, and $z$-band images where the two components are not as well resolved as in the other bands, we fixed the offset between the two components to that determined from the Keck AO image. The deblended $K$-band fluxes from the UKIDSS image agree within $1 \sigma$ with those measured from the AO image. We measured all fluxes with a $3^{\prime \prime}$ radius aperture centered between the primary and the secondary. We chose this aperture because it includes most of the flux and it agrees with the SDSS $r$-band Petrosian radius. The deblended photometry is tabulated in Table 1.

We fit the photometry using the empirical UV-to-mid-IR spectral energy distribution (SED) templates of Assef et al. (2010). Every object is modeled by a non-negative linear combination of three galaxy templates, corresponding to an old stellar population, a moderately star-forming galaxy and a starburst, in combination with a type-1 AGN template for which we allow for additional dust extinction (five free parameters). We fit the primary using the total FUV and NUV fluxes ${ }^{16}$ and the deblended SDSS and UKIDSS photometry, but using the WISE fluxes as upper bounds. As expected, the residual FUV and NUV fluxes from the fit are consistent with 0; so for the secondary, we use the $3 \sigma$ upper bounds in FUV and NUV based on their $1 \sigma$ errors. The best-fit primary SED can only account for part of the WISE fluxes; so for the secondary, we use the residual fluxes in the WISE bands. The SED fits of both components are shown in Figure 3.

\footnotetext{
16 Justified by the red optical SED of the secondary.
}

The best-fit SEDs are consistent with the type-1-type-2 AGN pair scenario. The AGN in the primary is slightly reddened $(E(B-V)=0.025)$. The secondary component requires a heavily reddened AGN $(E(B-V)=4.2)$ to match the blue 3.4-4.6 $\mu \mathrm{m}$ color that is indicative of warm dust. In the following, we use the best-fit SED models to derive the physical properties of the binary AGN. The quoted error bars of the luminosity/mass estimates discussed in the following sections are obtained after scaling the flux error bars to yield the best-fit SEDs with $\chi_{v}^{2}=1$.

\subsubsection{Black Hole and Host Galaxy Mass}

The black hole mass of the primary type-1 AGN is $\log \left(M_{\mathrm{BH}} / M_{\odot}\right)=8.06 \pm 0.24$ with FWHM $(\mathrm{H} \beta)=3800 \pm$ $100 \mathrm{~km} \mathrm{~s}^{-1}$ and $\nu L_{5100}=(9.5 \pm 3.0) \times 10^{43} \mathrm{erg} \mathrm{s}^{-1}$ (Equation (5) of Vestergaard \& Peterson 2006). The $\mathrm{H} \beta$ FWHM is from the SDSS spectrum. The $5100 \AA$ AGN luminosity is from the SED fit of the primary. The uncertainty in the black hole mass is dominated by the uncertainties in the virial coefficient and in the size-luminosity relation (Kaspi et al. 2005).

The best-fit SEDs imply stellar masses of $(1.7 \pm 0.3) \times$ $10^{11} M_{\odot}$ and $(2.4 \pm 0.4) \times 10^{11} M_{\odot}$ for the primary and secondary host galaxies, respectively, suggesting that SDSS J1502+1115 is close to an equal-mass merger. The stellar masses are estimated using the color-mass-to-light-ratio relation of Bell et al. (2003). The black hole mass and the host galaxy mass of the primary AGN is consistent with the Magorrian relation $\left(M_{\mathrm{BH}} / M_{\text {Bulge }} \simeq\right.$ 0.14\%; Häring \& Rix 2004; Magorrian et al. 1998). The Keck AO image indicates early-type morphologies for both host galaxies (Section 3.3).

\subsubsection{Black Hole Accretion Rate and Star Formation Rate}

We estimate black hole accretion rates from the AGN bolometric luminosities. Gallagher et al. (2007) argue that monochromatic $3 \mu \mathrm{m}$ luminosity is a more robust indicator of bolometric AGN luminosity than the conventional $5100 \AA$ luminosity because of its lack of sensitivity to dust extinction. The best-fit SEDs give AGN rest-frame $3 \mu \mathrm{m}$ luminosities of $v L_{v}(3 \mu \mathrm{m})=(1.6 \pm 0.5) \times 10^{44}$ and $(3.1 \pm 0.9) \times 10^{44} \mathrm{erg} \mathrm{s}^{-1}$ for the primary and secondary, respectively. Adopting the bolometric corrections from Hopkins et al. (2007), we get $L_{\text {bol }}=(1.7 \pm 0.5) \times 10^{45}$ and $(3.3 \pm 1.0) \times 10^{45} \mathrm{erg} \mathrm{s}^{-1}$. The total bolometric luminosity agrees well with the ROSAT soft X-ray derived bolometric luminosity, $L_{\text {bol }}=(5.2 \pm 1.8) \times 10^{45} \mathrm{erg} \mathrm{s}^{-1}$, using also the SED templates of Hopkins et al. (2007). The corresponding accretion rates are $\dot{M}_{\mathrm{BH}}=0.30 \pm 0.09$ and $0.58 \pm 0.17$ $(0.1 / \epsilon) M_{\odot} \mathrm{yr}^{-1}$, where $\epsilon$ is the radiative efficiency of the central engine. Assuming the same black hole to host galaxy mass ratio for the two components, the Eddington ratios are 0.12 and 0.16 for the primary and secondary, respectively, which are close to the mean Eddington ratios of optically selected AGNs at $z \sim 0.4$ (McLure \& Dunlop 2004).

Based on ultraviolet luminosities (Kennicutt 1998), the models give SFRs of $7 \pm 3$ and $8 \pm 1 M_{\odot} \mathrm{yr}^{-1}$ for the primary and secondary, respectively, consistent with [O II]-derived upper limits (Section 3.1).

\subsubsection{Radio Loudness}

Both AGNs in SDSS J1502+1115 are radio-moderate-toloud. The rest-frame $5 \mathrm{GHz}$ to $4400 \AA$ flux ratio of the primary gives a radio loudness of $R=9.8 \pm 2.9$, which is on the borderline between radio-moderate and radio-loud AGNs 
(Kellermann et al. 1989). The secondary AGN's optical emission is heavily obscured, and the extinction-corrected $4400 \AA$ flux of $0.18 \pm 0.05 \mathrm{mJy}$ implies $R=12.3 \pm 3.6$.

\subsection{Host Galaxy Morphology}

The primary appears essentially unresolved in the Keck AO image; it was used as the tip-tilt reference star for the LGSAO observations. There are no PSF reference stars within the field. However, we have found, in agreement with Carrasco et al. (2010), that the effective radius $r_{e}$ is not very sensitive to uncertainties in the PSF. We use a PSF derived from a well-exposed but unsaturated star in the field of another AGN, SDSS J131106.73+195234.2, which we observed on the same night. This star was the tip-tilt reference. It is slightly brighter (SDSS $r=17.0$ ) than SDSS J1502+1115 and the AO-corrected image has FWHM $=0$ !' 10 . Since the primary of SDSS J1502+1115 clearly has an unresolved component, we use an iterative process to disentangle the morphologies. We first convolve the PSF star with a Gaussian to match its FWHM to that of the Gaussian fit to the primary of SDSS J1502+1115, measured within a two-pixel (0'.08) radius. We use this modified PSF to model both components: the primary as a combination of a PSF and a Sérsic profile, and the secondary as a single Sérsic profile. We then use the output of this model to update the PSF FWHM and iterate. The final PSF FWHM is about 0.15 .

The unresolved component comprises $12_{-4}^{+8} \%$ of the total flux of the primary, roughly consistent with that from the SED fitting (24\% $\pm 9 \%$; Section 3.2). The primary host galaxy is significantly more compact than the secondary, with $r_{e}=$ $3.7 \pm 0.2 \mathrm{kpc}$, compared to the secondary's $r_{e}=6.5 \pm 0.6 \mathrm{kpc}$. The Sérsic indices for the primary and secondary host galaxies are $n=4.8 \pm 2.0$ and $2.9 \pm 0.2$, respectively. In spite of these differences, the masses and effective radii for both host galaxies are consistent with the mass-size relation of local early-type galaxies (Damjanov et al. 2009).

\section{SUMMARY}

SDSS J1502+1115 was initially identified as a candidate type-1-type-2 AGN pair by extensive AO imaging and integralfield spectroscopic follow-up of SDSS double-peaked AGNs. Here we have presented its EVLA images at 1.4, 5.0, and 8.5 GHz. These data reveal that each nucleus of the galaxies is associated with a steep spectrum compact radio source. The radio powers are inconsistent with star formation, indicating that the radio emission is powered by accretion. Therefore, the EVLA data confirm that SDSS J1502+1115 is a $7.4 \mathrm{kpc}$ separation type-1-type-2 AGN pair.

The AGNs appear to be normal Seyferts. SED fitting shows that SDSS J1502+1115 is a merger of two $\sim 10^{11} M_{\odot}$ galaxies. The accretion rates are 0.30 and $0.58(0.1 / \epsilon) M_{\odot} \mathrm{yr}^{-1}$ for the primary and the secondary, respectively. With both black hole masses around $10^{8} M_{\odot}$, the AGNs are accreting at Eddington ratios of $\sim 0.15$. With an angular separation of 1".4, future Chandra observations can provide spatially resolved X-ray imaging and spectroscopy of the binary.

It is surprising that both AGNs are close to radio loud ( $R \sim 10)$, because there are merely four binary AGN candidates from our survey (detection statistics consistent with that of
Tingay \& Wayth 2011). Finding one such object in four requires an extremely high radio-loud fraction, $50_{-10}^{+27} \%$, for AGNs in kiloparsec-scale binaries. A larger sample is clearly needed to further evaluate this result.

Z.Y.Z. acknowledges support by NSFC key project 10833006. A.D.M. is a research fellow of the Alexander von Humboldt Foundation of Germany. A.S. and S.G.D. were partially supported by NSF grants AST-0807900 and AST-0909182, respectively. S.G.D. also acknowledges support by the Ajax Foundation. The National Radio Astronomy Observatory is a facility of the National Science Foundation operated under cooperative agreement by Associated Universities, Inc.

Facilities: VLA, Keck:II (LGSAO/NIRC2), UH:2.2m (SNIFS), Sloan

\section{REFERENCES}

Aihara, H., Allende, P. C., An, D., et al. 2011, ApJS, 193, 29

Aldering, G., Adam, G., Antilogus, P., et al. 2002, Proc. SPIE, 4836, 61

Assef, R. J., Kochanek, C. S., Brodwin, M., et al. 2010, ApJ, 713, 970

Baldwin, J. A., Phillips, M. M., \& Terlevich, R. 1981, PASP, 93, 5

Barnes, J. E., \& Hernquist, L. 1996, ApJ, 471, 115

Bell, E. F., McIntosh, D. H., Katz, N., \& Weinberg, M. D. 2003, ApJS, 149, 289

Brusa, M., Zamorani, G., Comastri, A., et al. 2007, ApJS, 172, 353

Burke-Spolaor, S. 2011, MNRAS, 410, 2113

Cardelli, J. A., Clayton, G. C., \& Mathis, J. S. 1989, ApJ, 345, 245

Carrasco, E. R., Conselice, C. J., \& Trujillo, I. 2010, MNRAS, 405, 2253

Comerford, J. M., Gerke, B. F., Newman, J. A., et al. 2009, ApJ, 698, 956

Damjanov, I., McCarthy, P. J., Abraham, R. G., et al. 2009, ApJ, 695, 101

Fu, H., Myers, A. D., Djorgovski, S. G., \& Yan, L. 2011a, ApJ, 733, 103

Fu, H., Yan, L., Myers, A. D., et al. 2011b, arXiv:1107.3564

Gallagher, S. C., Richards, G. T., Lacy, M., et al. 2007, ApJ, 661, 30

Gerke, B. F., Newman, J. A., Lotz, J., et al. 2007, ApJ, 660, L23

Häring, N., \& Rix, H.-W. 2004, ApJ, 604, L89

Hennawi, J. F., Myers, A. D., Shen, Y., et al. 2010, ApJ, 719, 1672

Ho, L. C. 2008, ARA\&A, 46, 475

Hopkins, P. F., Richards, G. T., \& Hernquist, L. 2007, ApJ, 654, 731

Junkkarinen, V., Shields, G. A., Beaver, E. A., et al. 2001, ApJ, 549, L155

Kaspi, S., Maoz, D., Netzer, H., et al. 2005, ApJ, 629, 61

Kellermann, K. I., Sramek, R., Schmidt, M., Shaffer, D. B., \& Green, R 1989, AJ, 98, 1195

Kennicutt, R. C. J. 1998, ARA\&A, 36, 189

Komossa, S., Burwitz, V., Hasinger, G., et al. 2003, ApJ, 582, L15

Koss, M., Mushotzky, R., Treister, E., et al. 2011, ApJ, 735, L42

Lawrence, A., Warren, S. J., Almaini, O., et al. 2007, MNRAS, 379, 1599

Liu, X., Greene, J. E., Shen, Y., \& Strauss, M. A. 2010a, ApJ, 715, L30

Liu, X., Shen, Y., Strauss, M. A., \& Greene, J. E. 2010b, ApJ, 708, 427

Liu, X., Shen, Y., Strauss, M. A., \& Hao, L. 2011, ApJ, 737, 101

Magorrian, J., Tremaine, S., Richstone, D., et al. 1998, AJ, 115, 2285

Martin, D. C., Fanson, J., Schiminovich, D., et al. 2005, ApJ, 619, L1

McGurk, R. C., Max, C. E., Rosario, D. J., et al. 2011, ApJ, 738, L2

McLure, R. J., \& Dunlop, J. S. 2004, MNRAS, 352, 1390

Myers, A. D., Richards, G. T., Brunner, R. J., et al. 2008, ApJ, 678, 635

O'Dea, C. P. 1998, PASP, 110, 493

Osterbrock, D. E., \& Ferland, G. J. 2006, Astrophysics of Gaseous Nebulae and Active Galactic Nuclei (Sausalito, CA: Univ. Science Books)

Peng, C. Y., Ho, L. C., Impey, C. D., \& Rix, H.-W. 2010, AJ, 139, 2097

Perley, R. A., Chandler, C. J., Butler, B. J., \& Wrobel, J. M. 2011, ApJ, 739, L1

Richstone, D., Ajhar, E. A., Bender, R., et al. 1998, Nature, 395, 14

Shen, Y., Liu, X., Greene, J. E., \& Strauss, M. A. 2011, ApJ, 735, 48

Smith, K. L., Shields, G. A., Bonning, E. W., et al. 2010, ApJ, 716, 866

Tingay, S. J., \& Wayth, R. B. 2011, AJ, 141, 174

Vestergaard, M., \& Peterson, B. M. 2006, ApJ, 641, 689

Wang, J.-M., Chen, Y.-M., Hu, C., et al. 2009, ApJ, 705, L76

White, R. L., Becker, R. H., Helfand, D. J., \& Gregg, M. D. 1997, ApJ, 475, 479

Wizinowich, P. L., Le Mignant, D., Bouchez, A. H., et al. 2006, PASP, 118, 297

Wright, E. L., Eisenhardt, P. R. M., Mainzer, A. K., et al. 2010, AJ, 140, 1868

Yun, M. S., Reddy, N. A., \& Condon, J. J. 2001, ApJ, 554, 803 